\title{
Téoros
}

Revue de recherche en tourisme

\section{Entre inertie et dynamisme touristique}

\section{Le cas parisien}

\section{Édith Fagnoni et Colette Aymard}

Volume 21, numéro 1, printemps 2002

Les villes capitales

URI : https://id.erudit.org/iderudit/1071532ar

DOI : https://doi.org/10.7202/1071532ar

Aller au sommaire du numéro

Éditeur(s)

Université du Québec à Montréal

ISSN

0712-8657 (imprimé)

1923-2705 (numérique)

Découvrir la revue

Citer cet article

Fagnoni, É. \& Aymard, C. (2002). Entre inertie et dynamisme touristique : le cas parisien. Téoros, 21(1), 4-11. https://doi.org/10.7202/1071532ar d'utilisation que vous pouvez consulter en ligne.

https://apropos.erudit.org/fr/usagers/politique-dutilisation/ 


\section{Entre inertie et
dynamisme touristique \\ le cas parisien}

\section{Édith Fagnoni et Colette Aymard}

P aris, la force expressive de son nom évoque d'emblée le rayonnement international de la capitale auquel participe la réalité économique du tourisme parisien : 26 millions de visiteurs par an, répartis en 16 millions d'étrangers et 10 millions de français ; et 10 millions de visiteurs supplémentaires pour l'Ile de France dans sa globalité. Sans conteste, l'image touristique parisienne s'est construite autour de toute la symbolique urbaine de la capitale : les monuments. Pôle international du tourisme (131 000 emplois salariés directs, soit $10 \%$ des emplois de Paris, près de 15 \% de l'emploi touristique national et environ 9 milliards d'euros de retombées économiques), Paris exerce une fascination certaine. Il en résulte un tropisme parisien établi, confortant le rôle de métropolisation touristique de Paris, et l'image d'une ville prestigieuse. Paris, pour ses fonctions politiques, économiques, financières, culturelles, intellectuelles, possède des équipements de premier plan pour le tourisme, en particulier des réseaux de communication denses et efficaces, et une hôtellerie diversifiée et de grande capacité (voit Téoros article de G. Cazes dans ce même numéro).

La réflexion sur les nouveaux temps sociaux portée par la dilution du travail dans le temps, associée à l'augmentation du volume de temps libre et à la bataille de la vitesse, conforte la politique des courts séjours et implique de nouveaux rapports à l'espace. Cette rapide et profonde modification des comportements en matière de consommation touristique a ouvert au tourisme urbain de réelles perspectives de redéveloppement. Le nouveau rapport à la mobilité a permis de renouer des liens entre le tourisme et la ville, alors que la massification du tourisme avait eu tendanè à considérer la ville comme seul pôle émetteur de touristes. Les métropoles se repositionnent ainsi dans leur fonction réceptrice. La ville est devenue la première destination du tourisme de courte durée ; elle s'affirme comme territoire spécifique de consommation touristique et devient destination à part entière. De plus, on soulignera la non-saisonnalité du tourisme urbain, résultant d'une complémentarité temporelle entre tourisme d'affaires hors période estivale et en semaine, et tourisme d'agrếment durant les week-ends et les vacances.

L'objet de cette réflexion n'est pas de confirmer le rôle touristique des villes. mais de vérifier ce rôle de ville-destination à travers l'exemple de Paris. La notoriété et l'identité de la capitale repose sur un patrimoine social et culturel accumulé au cours de l'histoire, et apparaît d'emblée sur le plan touristique comme une ville-destination. Mais dans un contexte de concurrence accrue - Paris qui Evoque à la fois l'héritage d'une France autocentréc et une métropole de plus en plus ouverte sur l'Europe - l'évidence de la destination touristique de Paris ne suffit plus. De plus, les attentats du 11 septembre 2001 ont souligné la vulnérabilité du secteur touristique. Comment satisfaire et fidéliser des clientèles au comportement varié ? Comment dynamiser l'image du Paris traditionnel qui s'oppose désormais à un tourisme multi-facettes ? Comment rattacher à l'aire parisienne les nouvelles formes touristiques du XXI" siècle, désormais incontournables, tel le parc d'attraction Disneyland Paris excentré dans l'Est parisien à Marne la Vallée ( 12 millions de visiteurs par an)? Comment dépasser, sans les nier, des images restées souvent figées, afin de conquérir de nouvelles clientèles, notamment les jeunes et les touristes en provenance de marchés émergents (Amérique du Sud, Asie (hors Japon), et Europe de l'Est)?

Dans le triptyque habituellement utilisé pour classer les formes de tourisme urbain (affaires, réunions, congrès; agrément, culture, distraction; autres motivations personnelles), nous limiterons le champ de l'analyse au tourisme de loisirs. Certes le tourisme d'affaires, qui englobe les secteurs distincts des congrès, des foires et salons, des voyages de motivation (appelés incentives), des séminaires d'entreprises et des réunions d'affaires, est un des piliers du tourisme urbain en général et plus particulièrement à Paris ; la capitale occupe pour la vingt deuxième année consécutive la première place mondiale en matière de congrès. Mais c'est le tourisme de loisirs qui se nourrit le plus des stéréotypes de Paris et forme le gros des bataillons de touristes fréquentant la capitale. Nous vérifierons donc comment les politiques tendent à développer le tourisme de loisirs en terme d'attraction, d'accueil, voire de fidélisation.

\section{Splendeur et clichés d'une ville-musée}

Si Paris est devenu le premier centre touristique de France, il le doit en premier lieu 
à son patrimoine architectural et culturel. Les siècles ont façonné Paris. Il en résulte des images phares bien connues: Paris ancienne Lutèce, aujourd'hui capitale politique et intellectuelle ; Paris avec ses îles, île de la Cité ou île Saint-Louis ; Paris sur Seine, avee ses ponts ; Paris port fluvial ; Paris avec ses collines, Montmartre, Ménilmontant, Sainte-Geneviève; Paris universités ; Paris musées : Paris monuments; Paris luxe ; Paris flonflon ; Paris gastronomie ; Paris shopping "Paris night and day. Le touriste pourra, à sa guise. puiser dans la polyvalence thematique de Paris les prétextes de sa visite et perpétuer le sentiment d'une éternelle découverte, voire redecouverte. C'est un Paris infini aux multiples facettes et aux multiples parcours. Les arguments ne manquent pas pour faire de cette métropole un pôle touristique incontournable. Paris a donné naissance et continue à donner naissance à des mythes de portée universelle.

Le développement du tourisme à Paris s'est fait spontanement par le seul rayonnement international de la capitale. Les forces vives du tourisme parisien sont liées aux

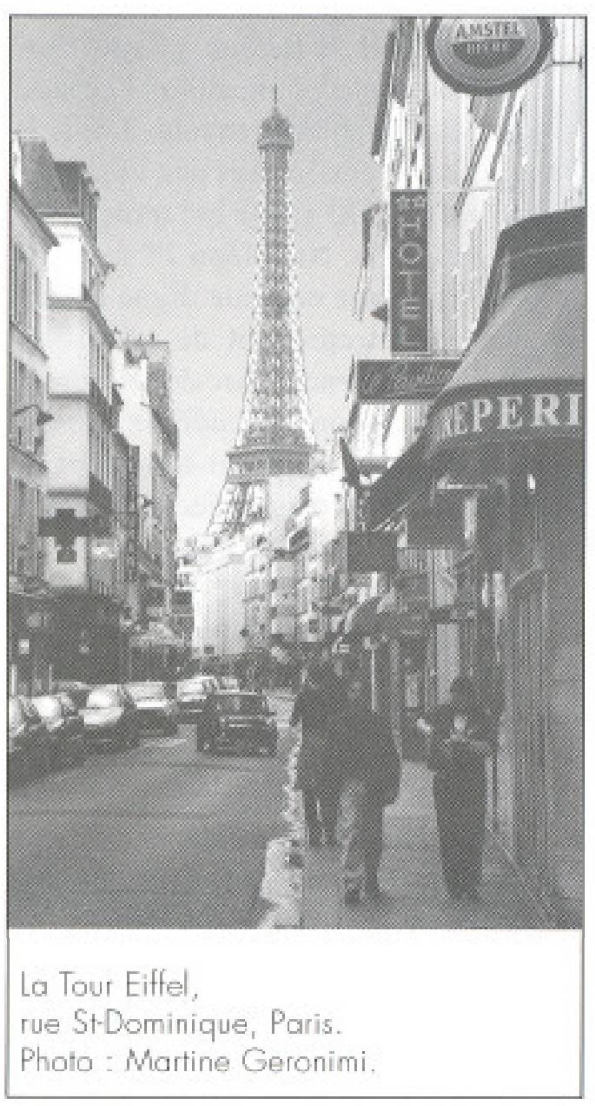

actions et aux stratégies cumulées qui ont contribué à construire et à consolider sa vocation de capitale politique, économique et culturelle. Paris a traversé l'Histoire, s'est transformé voire embelli et profite désormais de son patrimoine. C'est pourquoi il ne s'agit pas de passer en revue toute l'histoire de Paris, mais de comprendre l'importance des images qui agissent sur l'imaginaire du visiteur.

\section{La Seine, un défilé̉ architectural}

La promenade le long de la Seine est un parcours obligé du touriste venant pour la première fois a Paris. Elle lui permet de traverser la ville et d'apercevoir un panorama aussi varié qu'imposant. La majorité des monuments de Paris se trouvent le long de la Seine ou dans ses environs immédiats. Il semble qu'aucune autre cité européenne ne se définisse à ce point par son fleuve. La relation qui l'unit à Paris en fait l'axe de référence de la ville. Le visiteur s'émerveille devant l'île de la Cité, ancien village de pecheurs celtes, les Parisii, conquis par les Romains. Peu de vestiges de cette époque subsistent, si ce n'est sur la rive gauche de la Seine, avec les thermes de Cluny et les arenes de Lutece, Cette île demeure l'âme historique du développenent géographique de Paris, C'est à partir de ce lieu qu'au Moyen Âge les Capétiens décident d'agrandir la capitale. L'île de la Cité devient alors siège de l'autorité politique et religieuse. Le visiteur remarque la concentration des chefs-d'cuvre du Moyen Age, tels lä cathédrale Notre-Dame, l'ancien Palais= Royal désormais Palais de Justice, et la Conciergerie dont les tours témoignent de sa transformation en prison sous la Révolution française. Tout au long de son parcours, le visiteur est sensible aux nombreux ponts qui enjambent la Seine, en particulier le Pont-Neuf, premier pont de pierre sans habitation, ou encore le pont des Arts, construit en fonte. Plus à l'ouest, le touriste longe, sur la rive droite de la Seine, le Louvre, joyau muséal mondial, dont la façade monumentale souligne la force du pouvoir des souverains de lä Renaissance, perpétuée par les multiples transformations au cours des siècles: l'arc de triomphe du Carrousel celebre les victoires de Napo- léon $1^{\text {er }}$ et la pyramide de verié éclaire de la lumiere du $\mathrm{XX}$ siècle les collections du musée. Le visiteur découvre, sur la rive gauche, le musée d'Orsay, réhabilitation de $\mathrm{l}^{\dagger}$ ancienne gare du XIXe sibcle qui abrite depuis 1986 des collections artistiques couvrant la période 1848-1914, période au cours de laquelle on retient symboliquement les cuvres impressionnistes qui ont établi sa réputation. Précisons que l'aspect industriel de la gare (verre et métal) a été volontairement caché à l'extérieur par une façade monumentale inspirée du Louvie, afin de satisfaire aux exigences esthétiques de cet élégant quartier. La passerelle piétonnière de Solférino le relie au jardin des Tuileries sur la rive droite. Un cadre de nature, redessiné par Le Nôtre qui en ă fait un chef-d'auvre classique, complete la vic elégante. Le visiteur arrive alors au PalaisBourbon (actuelle Assemblee nationale), édifice du XVII" ${ }^{\circ}$ siecle regardant vers la place de la Concorde. Le touriste est frappé par ces témoignages d'une période historique particulièrement faste, s'étendant du $X V I^{e}$ à la fin du XVIII siecle. Puis, le parcours se poursuit avec le Pont Alexandre III et l'hôtel des Invalides rappelant I'importance du pouvoir militaire sous Louis XIV. Les dorures du dôme des Invalides et celles du pont Alexandre III attestent du rayonnement de la civilisation française.

Les témoignages architecturaux qui défilent ensuite devant le visiteur se rattachent à la fin du $X I X^{a}$ siècle et à la première partie du $X X$ siecle. La France \& éblouit $*$ par ses grandes expositions. L'Exposition universelle de 1900 lègue le Grand et le Petit Palais. Le visiteur découvre ensuite le palais de Tokyo (actuel musée d'Art moderne), un des édifices construit dans le style néoclassique pour l'Exposition universelle de 1937. Cet ensemble est complété, par l'aménagement du Trocadero avec le palais de Chaillot, aux deux immenses courbes dominant la Seine. La tour Eiffel, apogée de l'architecture de fer et symbole de popularité internationale de Paris, est Edifiée pour l'Exposition universelle de 1889. Sur la rive opposée, le visiteur passe devant la Maison de la Radio datant de 1963. Au pont de Grenelle, le touriste américain sourit devant la statue de la Liberté, tournée vers New York et 
ayant servi de modèle au statuaire Bartholdi pour réaliser la statue érigée dans la rade de New York. Le parcours se poursuit par la découverte du Front de Seine, témoignage de l'urbanisme parisien moderne, compose des seize tours de Grenelle. Cette promenade fluviale prépare la découverte du Paris a ville moderne $\%$, celui du $\mathrm{XXI}^{c}$ siècle et du quartier de La Défense avec ses 80 hectares de gratte-ciel.

Toutes les époques se côtoient dans ce défilé architectural en front de Seine, avec un seul objectif : l'affirmation de la fonction de capitale grâce à une monumentalité mise en spectacle. L'axe horizontal de la Seine s'oppose à la verticalité des architectures et concourt à donner une impression de majestueuse unité intemporelle. La Seine, a long fleuve tranquille $\%$, apporte à ce tableau, construit par l'homme, une douceur presque immatérielle. Le touriste trouve ici tous les clichés d'une ville romantique, avec ses ponts, ses îles et ses jardins. Les bouquinistes au bord de la Seine participent à l'élaboration de cette image d'une ville hors du temps. C'est cette splendeur, ce romantisme parisien, cette ville éternelle que louent tant d'artistes, peintres, Ecrivains ou chanteurs. Paris est la ville culturelle par excellence. L'Académie française et I'Institut de France éclairent de leur prestige la promenade du voyageur sensible à l'art et à la culture. Le défilé architectural en front de Seine présente au visiteur les grandes étapes de l'histoire de France et célèbre Paris-berceau de la France, Paris-ville historique par excellence. Le visiteur saisit la volontế des pouvoirs politiques de rattacher culturellement la capitale à l'histoire universelle et à ses grands symboles: l'Obélisque, symbole de l'alliance entre le cosmos et la terre, témoin des premiers idéogrammes et des progrès de la connaissance : le génie ailé de la Bastille, célébrant la fin de l'autocratie et l'envolée des Droits de l'homme ; la statue de la Liberté a miniaturisee $*$ affirmant la victoire des grands combats idéologiques et le lien indéfectible entre ancien et nouveau monde. $\mathrm{Ce}$ Paris immuable est celui des prospectus publicitaires. C'est le Paris * cliché qu" attend le touriste. C'est le Paris monumental figé le long de la Seine.

\section{Une ville toujours en mouvement entre unité et multiplicité}

Le rapport du territoire au temps est au cour de cette découverte anachronique de lá monumentalité de Paris, qui puise ses sources dans les différents cycles historiques, correspondant tour à tour à des sommets d'expansion b́conomique, de centralisation politique, de certitude technique, de rupture et de récession. La métropolisation grandissante marque l'espace. La ville évolue et, à chaque période, les monuments ponctuent le temps et le territoire urbain. Ils construisent l' identité de la ville. Paris s'accroche à ses monuments, pôles d'attraction dans les quartiers qui participent à l'élaboration d'une entité touristique. Cette juxtaposition des différents héritages traver'sés par le touriste le long de la Seine illustre la nécessité de la ville d'évoluer avec son temps, de moderniser ses quartiers, afin qu'ils répondent à l'augmentation démographique et à l'évolution des fonctions. Paris a toujours eu le souci d'établir une cohérence entre patrimoine monumental et urbanisme. Le développement de la ville rêlègue alors la Seine à un axe monumental et Paris se détache du fleuve pour sans cesse s'étendre. Après 1945, l'évolution architecturale de Paris ressemble à celle de beaucoup d'autres villes : des tours et des barres massives et des immeubles très élaborés : I'Unesco, le C.N.I.T. (Centre de Congrès et d'Expositions), la tour Montparnasse, le Palais des Congrès. De nouveaux monuments sont érigés et leurs spécificités architecturales marquent le paysage urbain. Les présidents de la $\mathrm{V}^{\mathrm{e}}$ République ont largement contribué à agrandir le patrimoine monumental parisien: le centre Georges-Pompidou, le Grand Louvre, 1'Institut du Monde Arabe, l'Opéra Bastille, la Grande Bibliotheque ou encore le Ministère de l'Économie et des Finances, en passant par la reconversion des abattoirs de la Villette en Cité des Sciences et de l'Industrie. Des formes nouvelles s'insèrent dans le paysage existant. C'est dans cette alliance du passé et du futur que le touriste puise et repuise les prétextes de sa visite. L'art architectural s'élargit et il devient, à part entière, élément d'urbanisme et de paysage; les édifices sont de plus en plus conçus comme les éléments d"un vaste ensemble : des quartiers sont préservés, d'autres remodelés. Paris est une ville sans cesse en mouvement, s'affichant entre patrimoine et modernité.

L'articulation entre la ville intemporelle et la ville en bouleversement passe par cette mise en perspective de l'espace urbain. Les perspectives contribuent à magnifier le pouvoir, à créer une capitale, à unifier le Paris de toutes les époques. Très tôt existe une réflexion sur l'aménagement du territoire à l'échelle urbaine. L'origine de cette mise en perspective de Paris remonte essentiellement au Grand siècle : les Bourbons - en élevant au plus haut point la monarchie absolue et centralisatrice - encouragent l'embellissement de la ville. Outre le fait de se parer de nombreux édifices, Paris se dote d'axes de circulation et d'un nouveau type de places géométriques avec la place Royale (actuelle place des Vosges) ou encore la place Dauphine. De nouvelles fortifications rive droite (actuels Grands Boulevards) permettent à la ville de s'agrandir ; de nouveaux quartiers apparaissent dans le faubourg Saint-Honoré, l'île SaintLouis, le Marais, le faubourg Saint-Germain. D'autres places organisent l'espace, a l'exemple des places royales Louis le Grand (place Vendôme) et des Victoires. Mais c'est le XIX ${ }^{\circ}$ siècle qui transforme réellement Paris. Napoléon $1^{\text {cr }}$ souhaite faire de Paris une capitale digne de son Empire en entreprenant de véritables projets : il commence l'Arc de Triomphe, la Bourse, la colonne Vendôme, les canaux de l'Ourcq, de Saint-Martin et de Saint-Denis. Mais c'est le second Empire qui transforme véritablement Paris et lui donne sa physionomie actuelle. Napoléon III avait confié au Baron Haussmann la direction des travaux. L'objectif était de construire un Paris grande capitale moderne, adapté aux transports modernes (construction des gares), assaini et aéré de jardins (2 000 hectares de pares et de jardins, depuis les bois de Boulogne et de Vincennes jusqu'aux petits squares, en passant par les pares des Buttes-Chaumont et de Montsouris). Haussmann a aéra * Paris en implantant des places d'où rayonnent de grandes 
percées, telles la place de l'Étoile, la place du Châtelet ou encore la place de l'Opéra Garnier. Le touriste, empruntant ces grands boulevards, bordés d'arbres et d'immeubles en pierre de taille selon une architecture prédéfinie et uniforme, a l'impression de parcourir une unité architecturale malgré la diversité intérieute des quartiers. Les places constituent des articulations entre les différents axes, quartiers et monuments et créent des perspectives magnifiant l'urbanisme parisien. De cette époque date le système d'égouts à Paris, autre curiosité de la ville. La Grande Arche participe à la construction des perspectives : nième porte de Paris, elle relie le XXI ${ }^{\circ}$ siècle à Napolénon et au Moyen Âge. Elle termine la plus longue perspective du monde, depuis le Louvre en passant par la place de la Concorde, les Champs-Élysées et l'Arc de Triomphe. Elle est la clé de voûte de La Défense et prolonge de quelques kilomètres l'ancien axe triomphal parisien. L'évolution de la ville profite à son image touristique.

Paris est aussi traditionnellement la capitale des plaisirs et de la mode. De son héritage d'artisans maîtres d'œuvre, tels que menuisiers et ébénistes du faubourg Saint-Antoine, façonniers du textile des rues Saint-Martin et Saint-Denis, elle a su forger sa domination dans les secteurs de la haute couture, de la bijouterie-joaillerie et des industries du luxe. A l'image de l'électricité qui, dès la fin du XIX siècle illumine les rues et les vitrines, Paris « éblouit *. Son rayonnement artistique. culturel et intellectuel est reconnu dans le monde entier. Les artistes viennent y vivre, s'y imprègnent des nouveaux courants et apportent leur talent. Paris, lieu de foisonnement culturel et de rencontres, est un passage obligé. D'importants mouvements artistiques naissent et se développent dans la capitale ; les peintres impressionnistes témoignent de cet avantgardisme. Pendant l'entre-deux-guerres, le rayonnement littéraire et artistique de Paris dépasse les frontières et les artistes de l'Europe entière affluent. L'Exposition internationale des Arts décoratifs de 1925 lance le style Art déco et marque un moment exceptionnel des années folles. Le Corbusier bouleverse l'architecture moderne avec l'utilisation de l'acier et du béton et l'introduction de formes géométriques. Des années 1920 aux années 1940, Paris attire peintres, musiciens, écrivains et cinéastes venus du monde entier. $\mathrm{Pi}-$ casso, Braque, Man Ray fondent des écoles artistiques. Pour les touristes, Paris demeure, à travers ses musées, ses galeries, ses spectacles et ses concerts, une véritable capitale culturelle avant-gardiste et festive.

Le touriste peut allier flânerie historique ou architecturale au tourisme culturel, ou encore au shopping. Paris est une ville d'affaires, une ville marchande, une métropole attractive par ses fonctions très diverses tant en quantité qu'en qualité. Cette ville sait se distinguer par la qualité de ses divertissements. La gamme des spectacles s'étend du café-théâtre à l'Opéra, en passant par les spectacles de variétés et les concerts. De mềne, le touriste peut aussi bien découvrir les artistespeintres de Montmartre que les galeries de Saint-Germain-des-Prés ou encore les expositions branchées de Belleville. Paris est une ville multiple proposant toute la gamme des divertissements. C'est la capitale des loisirs et de la mode. Paris est une ville a lumière s qui se met sans cesse au goût du jour pour développer et amplifier son attraction.

\section{Des images fígées à dépasser}

Mais cette ville, toujours en mouvement, ne restitue-t-elle pas des images bien souvent figées? C'est dans ce Paris historique que la ville forge ses images touristiques. Le parcours touristique le long du fleuve confirme bien que la Seine est la matrice de la ville. Les monuments qui la jalonnent sont la marque d'un pouvoir centralisateur qui a fait de Paris sa capitale et le symbole de sa puissance. La généralisation des pratiques touristiques a done bénéficié de cette longue construction historique. Paris s'est imposé comme destination touristique, mais l'image du Paris traditionnel ne s'est pas construite avec le tourisme pour priorité. L'organisation et la construction du Paris monumental, culturel et urbanistique sont le résultat d'une ville opulente, capitale d'une nation riche d'un passé politique, industriel et colonial. $\mathrm{C}^{+}$est surtout le XIXe et la première partie du $X X^{e}$ siècles qui ont donné à Paris ses images phares actuelles : la ville Lumière, symbole de beauté, d'élégance et de plaisirs est née de la « révolution haussmannienne s, avec l'apparition du tourisme moderne ou du tourisme de consommateurs de plaisirs. Or, ces images sont nées sans que l'on ait cherchè à développer une spécificité touristique. Seules les Expositions universelles, vitrines de la France et de son * empire *, ont êt animées d'une certaine stratégie touristique. Le second Empire et les années qui ont suivi ont en quelque sorte donné à Paris les grandes lignes de sa physionomie actuelle. L'opérette \& La Vie Parisienne s (1866) d'Offenbach reflète cette joie de vivre du second Empire. L'image de la * ville plaisir * - notamment autour des grands cabarets parisiens - se développe. On fixe à cette époque les stéréotypes de Paris. D'autres capitales européennes forgent leur image sur le même modèle, en particulier Vienne qui puise son image dans la grandeur de l'Empire austro-hongrois. Alors qu'à l'époque c'était une réalitê, aujourd'hui il s'agit d'une exploitation du passé ; on vit sur des acquis. Ce Paris aux représentations figées existe. Il attire de nombreux visiteurs. Au fond, ce sont des lieux, des atmosphères, des traditions et des modes de vie (a l'exemple du bistrot parisien) qui se sont codifiés et qui sont devenus des produits touristiques. Aujourd'hui, ces clichés habituels sont ceux que propose le touropérateur. Ils s'appuient sur des territorialités touristiques bien précises, des points de passage obligés, lesquels sont nourris d'images, à l'exemple du Poulbot de Montmartre ou des bouquinistes le long de la Seine. L'articulation touristique s'élabore autour des monuments et du fleuve, lequel contribue à donner une vue stéréotypée du Paris historique. Cette vision « clichée * de Paris doit se perpétuer ; elle est souvent la raison d'une première visite à Paris. Mais parle-t-elle aux jeunes générations (techno, gane boy et autres cybermania) peu sensibles à l'histoire et victimes du nivellement culturel sous influence americaine ? Y-a-t-il des perspectives autres que le tourisme tradi- 
tionnel ? Comment renforcer et diversifier l'attrait du tourisme parisien ? Comment fortifier le tourisme parisien ? Dans un climat a la fois de concurrence et de mobilité accrues, il convient de fidéliser la clientèle, mais aussi de capter de nouvelles clientêles. Comment séduire de nouvelles cibles ? Lévidence de la destination touristique de Paris ne suffit plus. Il y a aujourd hui un réel besoin de renouvellement. Comment renouveler l'offre touristique de la ville la plus visitée au monde? Si Paris est une capitale touristique, c'est-à-dire une ville phare de l'activité touristique, elle est confrontée à des problemes d'attraction, de modernisation de son image et de renouvellement de ses potentialités. Or les stéréotypes n'ont guère évolué. Le tourisme s'est donc codifié et souvent uniformisé et, faute d'avoir su se renouveler, s'est contenté $\mathrm{d}^{+}$exploiter jusqu'à épuisement les stếréotypes du passé. Face à cet immobilisme touristique, de véritables stratégies de développement touristiques s'imposent. Paris a besoin d'innover.

\section{De la nécessité d'une stratégie de communication sur l'image}

Si Paris a étonné le monde au $\mathrm{XIX} \mathrm{X}^{\mathrm{s}}$ siècle, il ne s"agit pas de faire la promotion d'un lieu de pèlerinage. On peut se demander si le poids de l'héritage, atout certain du tourisme parisien, n'est pas devenu, dans une certaine mesure, un handicap à son renouvellement. Il semble, depuis une quinzaine d'années, que les préoccupations touristiques de Paris aient été guidées par une politique de mise à niveau des structures anciennes. Paris recycle son passé au goût du jour. Cette stratégie passe par divers ajustements : on repense les modalités d'accueil, l'accès au monument et à l'équipement, à l'exemple du réaménagement đu Grand Louvre doté d'un pôle central d'accueil, d' un espace commerçant et d'un espace restauration, ce qui en fait aujourd'hui l'un des musées les plus modernes au monde ; ou encore du Palais des Congrès, où a été repensé l'accueil du tourisme d'affaires. L'annonce récente de la mise en chantier de la tour Eiffel s' inscrit dans cette même optique : améliorer l'accueil des 6 millions de visiteurs en créant en sous-sol un accès centralisé autour de la zone de la billetterie, associé à l'aménagement d'une salle de conférences, de congrès et de spectacles, à des restaurants et à une zone de chalandise. Par ailleurs, un espace destiné à la vente de produits dérivés manque actuellement. Ce souci de mise à niveau des structures anciennes passe donc par une phase de $\alpha$ relifting * ; il s'agit de mieux accueillir le client pour le garder plus longtemps et le pousser à la consommation. Il en est de même pour un grand nombre de palaces parisiens.

Le rayonnement du tourisme parisien passe aussi par les établissements hôteliers de renommée, du Ritz au Georges V, en passant par le Crillon ou le Grand Hôtel Intercontinental. De réhabilitation en création (implantation d"Hyatt en 1997), l'hôtellerie de grand luxe contribue à la célébrité de Paris et participe à l'attractivité de la capitale. Les salles de concert, notamment l'Olympia, et les thêatres ont connu la même stratégie de remise au goût du jour. Paris reste une vitrine de la France, une vitrine qui se modernise, certes, tout en gardant des fonctions de prestige qui lui valent son titre de ville de luxe. Paris demeure une ville qui suscite l'emerveillement, digne d'un conte de fées. Monuments symboles, hôtellerie de luxe, avenues et places prestigieuses bordées de commerces de luxe - à l'exemple de l'avenue Montaigne, de la rue du Faubourg Saint-Honoré ou encore de la place Vendôme - renvoient à l'image de la haute couture associée aux noms de Coco Chanel, de Christian Dior ou d'Yves SaintLaurent. Ces stéréotypes sont exploités jusqu'à épuisement sans réellement renouveler ni les images, ni les lieux. La politique monumentale, qui se posait jusqu'alors en autosuffisance touristique, ne suffit plus pour capter de nouvelles clientèles. De plus, face à l'européanisation à grande vitesse, Paris, certes, attire les regards et les flux, mais se trouve de plus en plus en compétition avec d'autres villes (Londres, Berlin, Vienne). Paris a donc besoin d' innover et de développer d'autres facteurs d'attractivité urbaine, renvoyant à des images nouvelles et à une territorialité touristique davantage éclatée. Au couur des stratégies : la communication, le tourisme des jeunes, le rapprochement entre les Parisiens et les touristes et le développement de l'accueil. D'oủ la nécessité d'une stratëgie de communication sur l'image, après avoir récusé les atouts et défini de nouveaux pôles d'attraction.

\section{Créer des êvènements porteurs}

Face à ce Paris en voie de « ringardisation *, il s'agit aujourd'hui d'apporter un contre-pied à l'image du Paris touristique traditionnel, sans cependant le nier. Paris - ville monumentale, ville d'effervescence créative, ville de pouvoir - doit être remise à la mode. L'atout du tourisme urbain étant sa pratique tout au long de l'année, Paris souhaite devenir la ville du * happening permanent . Mais comment ancrer Paris dans la modernité ? Une nouvelle stratégie de communication, supposant des choix précis en matière $\mathrm{d}^{+}$identité parisienne, s'avère nécessaire. Construire cette communication autour de trois axes forts : le Paris \& convivial $\%$, celui des Parisiens, du Paris secret, du Paris insolite; le Paris " branché», celui des lieux à la mode, de l'effervescence des métropoles en général, du calendrier festif et événementiel, celui de la French touch ; et le Paris a carrefour mondial des cultures $\%$, celui du creuset de toutes les provinces de France et des nombreuses cultures étrangères. Ville de métissage culturel par excellence, Paris essaie de favoriser le brassage culturel, source de créativité Cette remise à la mode de Paris passe donc par de nouveaux lieux. Elle s'appuie sur la vitalité de nouveaux quartiers : Bercy Village, le quartier de la Grande Bibliothèque, le quartier Oberkampf ou encore le quartier du canal Saint-Martin. Dans le cadre du calendrier festif et événementiel, la politique de communication et de promotion s'appuie sur quelques événements : des animations festives estivales sont prévues le long des voies sur berges durant l'été 2002, notamment la mise de là ville à la plage (sable et palmiers devraient recréer cette atmosphère). Ce sont aussi les Rencontres de Musique Électronique dans la première quinzaine de septembre précédant la technoparade, la Nuit Blanche 
des 5 et 6 octobre, avec l'ouverture et la gratuité des édifices publics. S'ajoutent d'ores et déjà le championnat du monde d'athlétisme, prévu au stade de France en août 2003, le Congrès des Associations de Ville en 2004 et l'Exposition Internationale sur l'Image, qui se tiendra au pare du Bourget en 2004. L'attractivité de la ville a périodiquement besoin d'être valorisée. La dimension évènementielle, de plus en plus impulsée par la concurrence, répond à cette nécessité de mise en marchế et de création de nouveaux produits touristiques. L'objectif est d'arriver à faire de ces êvènements un motif de séjour touristique, ce qui exige un travail en amont de l'événement afin de le transformer en produit touristique. Les événements portés par la ville sont de plus en plus susceptibles d'intéresser les visiteurs ; courts séjours et réduction du temps de travail sont à l'origine de cette interaction grandissante. En 2001, la nouvelle équipe de la Mairie de Paris ayant mis en avant le thème de la qualité de vie, il paraît logique qu'il soit conjugué avec celui de la qualité de l'accueil lié au tourisme. Méthodologiquement, cette réflexion renvoie à un plan d'action transversal entre plan de communication de la ville et plan de communication touristique.

\section{S' ouvrir \% aux jeunes}

Les évènements retenus ciblent tous les types de clientèles, mais il faudrait s'intéresser plus particulièrement à la clientèle jeune. Ce tourisme de jeunes représente $15 \%$ des 26 millions de touristes venus à Paris en 2000, soit 4 millions de personnes par an. En comparaison avec des capitales proches, comme Londres ou Berlin, le public des jeunes n'avait guère été retenu jusqu' alors comme public prioritaire à Paris, car il est souvent perçu comme une clientèle uniforme au faible pouvoir d'achat. Développer le tourisme des jeunes nécessite, de plus, une politique musclée d'adaptation de l'hébergement. Avec une capacité de 1300 chambres et de 520 emplacements de campingcaravaning (selon l'UNAT - I'Union Nationale des Associations de Tourisme et de plein air), Paris manque cruellement d'hébergements adaptés aux jeunes, Outre l'hébergement, il paraît nécessaire d'aménager des espaces collectifs orientés vers les centres d'intérêt des jeunes : la pratique de loisirs sportifs - en particulier la glisse, la musique, l'expression artistique et l'utilisation des nouvelles technologies de l'information. Des partenariats doivent être mis en place avec des professionnels spécialisés dans les loisirs des jeunes.

L'espace du Paris intra-muros étant de plus en plus limité, c'est probablement dans la périphérie immédiate que ces projets devront se réaliser. Cette approche renvoie au souci de sortir le Paris touristique de ses limites spatiales. Il semble que la construction d'une nouvelle image doit être menée en interaction avec les espaces limitrophes, espaces potentiellement touristiques de la capitale. Il s'agit de construire des activités réciproques entre $\mathrm{Pa}$ ris et sa proche banlieue. A titre d'exemple, le cas de la ville de Saint-Denis, en malêtre d'images, laisse aujourd'hui émerger une vocation touristique qui, outre la Basilique, se précise autour du Stade de France avec le tourisme sportif. Les nombreuses industries souhaitent aussi profiter du développement récent du tourisme industriel. Cette " périphérisation » touristique permettrait de transformer la proche banlieue en nouvel espace d'accueil. Les communes concernées pourraient alors bénéficier d'une certaine sédentarisation touristique. D. Pearce, dans son analyse spatiale sur le tourisme à Paris, insiste sur cette transformation de sites ponctuels de la banlieue en espace d'accueil : ne pas les limiter à un tourisme de passage, mais leur donner la dimension d'un espace d'accueil. Cette nécessaire articulation spatiale entre les différents projets touristiques demeure, bien évidemment, subordonnée à la collaboration entre les élus. La petite couronne de Paris peut compléter, voire renouveler l'offre touristique de la capitale, participant ainsi au processus de métropolisation touristique.

Cibler la clientèle jeune permettrait d'empêcher la sclérose de Paris en « villemusée * et d'innover en favorisant les échanges, les évềnements et la cré́ation. Or, créer des animations pour développer une image plus dynamique signifie un nécessaire rapprochement entre Parisiens et visiteurs.

\section{Tourisme et vie permanente : un territoire à partager}

L'activité touristique bénéficie des opérations $\mathrm{d}^{+}$amélioration urbaine en faveur des Parisiens. L'embellissement de l'habitat avec le ravalement des façades, mais aussi la «piétonisation * de certains espaces, en sont des exemples : espaces piétonniers plus nombreux et trottoirs plus larges, à l'exemple du réaménagement des Champs-Élysées ; tout comme la multiplication des centres ou des galeries marchandes, tels les nombreuses galeries sur les Champs-Élysées, le forum des Halles, le réaménagement des Trois Quartiers à la Madeleine, la Défense avec les Quatre Temps, Saint-Lazare et le passage du Havre ou encore le centre commercial place d'Italie. Dans ces espaces, le visiteur se confond avec le Parisien. Il vit la vie du Parisien et Paris en profite pour relancer le tourisme lié au shopping qui se décline, selon les lieux, à la fois en tourisme de shopping haut de gamme (avenue Montaigne, rue Saint-Honoré, avenue des Champs-Élysées, place Vendôme), en tourisme d'achats courants avec l'impor= tance des grands magasins de la capitale (du boulevard Haussmann au Bon Marehé ou à la Samaritaine), ou tout simplement en tourisme d'achats fortuits au hasard des rues et des quartiers qui invitent à déambuler (quartier du Marais, rue Saint-Paul, cours Saint-Émilion dans le nouveau Bercy). Les commerçants ont su s'adapter à la demande et aux différentes populations touristiques. L'avenue de l'Opéra cible les touristes japonais avec ses magasins hors taxes. Les puces et les grands antiquaires répondent à une demande d'authenticité, voire à une certaine nostalgie.

Le tourisme lié au shopping contribue à l'attraction de la ville et à sa réputation internationale. Les soldes sont de plus en plus un produit touristique.

Par ailleurs, la requalification d'espaces urbains permet de doter les villes, Paris en particulier, de nouveaux espaces résiden- 
tiels plurifonctionnels. Ces espaces regroupent des activités commerçantes, culturelles et de loisirs. Ce sont des espaces mixtes. Ils contribuent à la valorisation de l'image d'une ville. A Paris, cette a manière de refaire la ville m est particulièrement lisible dans l'Est parisien autour du réamenagement du viaduc des Arts, du quartier de la Bibliotheque Nationale de France et de Bercy-Village. L'ancien village du vin a laissé la place au nouveau quartier de Bercy : un parc de 70 hectares, nouvel espace de vie alliant logements, commerces, cinémas, établissements de restauration et de loisirs, profite au tourisme, tout en renforçant paradoxalement le sentiment identitaire des Parisiens. Un quartier des affaires s'est constitué autour de Bercy-Expo; le palais omnisports de Bercy accueille les plus grands spectacles et l'aménagement du parc de Bercy en huit jardins dits $k$ de mémoire $\%$ ponctue cette réhabilitation. La rénovation des pavillons permet $d^{+}$accueillir le musée des Arts forains, les Salons de musique et l'École de boulangerie et de pâtisserie de Paris. La requalification de ce quartier crée de fait aujourd' hui un espace polyvalent. Mais existe-t-il un réel souci touristique derrière la réorganisation d'espaces, tels que Bercy, ou encore derriêre la coulée verte, ou la politique des couloirs de bus parisiens? Le tourisme bénéficie des améliorations destinées à la vie des Parisiens; ces réalisations participent à la promotion de la ville et contribuent à sa nouvelle mise en image. Les caractéristiques de ces opérations montrent un double effet : economique, à partir de la réussite en termes d'attractivité et de revitalisation des espaces ; et politique, en utilisant la notion de projet urbain comme moteur du développement culturel pour valoriser l'image de certains quartiers et renforeer, de surcroit, l'image touristique. La conjugaison du tourisme et de la cul ture se révè le capitale pour la valorisation de la ville. La a multiculturalité $\%$, associée da la * polyfonctionnalite * du nouveau Bercy, plaide en faveur du rapprochement entre touristes et Parisiens et en faveur d'un redéploiement touristique spatial au bénéfice de découvertes a inédites $\%$. Le rôle du tourisme est capital pour l'image de la ville. De méme, lă culture profite au tourisme. Ces lieux culturels multiformes contribuent à la découverte par le touriste d'un Paris secret, voire insolite. Développer les liens entre les Parisiens et les vi= siteurs, grâce à des espaces communs, requiert que l'on se préoccupe de la politique d'accueil.

\section{Avoir le sens de l'accueil}

Le tourisme reste fondế sur la qualité de l'accueil. Mieux accueillir et mieux informer les visiteurs est un objectif incontournable qui doit préoccuper l'ensemble des institutionnels et professionnels du secteur. Le rôle que doit jouer un office de tourisme dans cette mission est fondamental. Dans le cas de Paris, on soulignera sa monosituation géographique sur les Champs-Élysées, paradoxe face a la territorialité touristique de plus en plus éclatée de la capitale. Diffuser l' information dans l'espace et dans le temps est un élément clé de la politique d"accueil et exige, d'une part, la multiplication des antennes de l'office de tourisme dans des lieux stratégiques, tels les gares et les aéroports, mais aussi dans des quartiers et sur les sites fortement visités. La réitêration et l'amplification d'une opération a ambassadeurs de l'accueil w testé avec succès sur le site de Notre-Dame durant l'été 2001 doit être menée pendant l'été 2002 sur sept ou huit autres sites: des comptoirs mobiles sont installés afin de pouvoir renseigner les touristes. D'autre part, il faudrait que l'information soit accessible 24 heures sur 24 , notamment en dehors des jours et des heures ouvrables, La politique Internet de l'office de tourisme de Paris auvre en ce sens ; elle a pour objectif de réaliser le passage de l'information à la réservation en moins de quatre clics.

En outre, ce rapport au temps soulève le probleme des horaires d'ouverture des édifices publics, en particulier des musées. Ils doivent s'adapter à un nouveau mode d'utilisation et de consommation du temps. La politique restrictive en vigueur, en matière d'horaire, ne correspond pas aux prétentions d'une ville qui se veut capitale culturelle universelle.
Par ailleurs, la réalisation d'une charte qualité, en partenariat avec les syndicats professionnels, serait un outil garant de la mise en auvre du label qualité et ce, dans des domaines variés, nommément:

les taxis parisiens, vêhiculant de nombreux touristes étrangers - ils doivent multiplier les efforts pour répondre de façon adaptée aux besoins et aux demandes de leurs clients (langue, serviabilite);

les cafés-restaurants - qui sont aussi, trop souvent, connus pour leurs problemes d'hygiene ;

ou encore les sites culturels - ils nécessitent une adaptabilité des commentaires aux differents objets selon différents niveaux de lecture et aux différentes nationalites des visiteurs.

Les transports représentent une autre composante de l'aceueil. Les cars de tourisme demeurent l'éternel problème de la capitale. La politique annoncée s'oriente vers la construction de pares de stationnement à la périphérie de Paris. Des solutions intermédiaires de a dépose/reprise a à proximité des zones d'hebergements hôteliers, corrélées à la création d'un systeme d'autocars tournant dans Paris intra-muros sont aussi envisagées. Une autre possibilité consisterait à mener une réflexion sur l'utilisation du fleuve. De plus, les nouveaux temps de la ville révèlent la nécessité d'adapter les transports en commun parisiens aux rythmes des usagers. En effet, avee la déstrueturation des temps sociaux, l'utilisation des transports s'est considérablement accrue le soir, réalité qui concerne autant les Parisiens que les touristes, ces derniers représentant $8 \%$ des utilisateurs de la RATP (Régie Autonome des Transports Parisiens). Louverture nocturne du métro doit être testée lors des manifestations festives des 21 juin, 14 juillet, 5 octobre (opération Nuit Blanche) et 31 décembre 2002. La fermeture des lignes à une heure du matin casse le rythme de la vie noeturne parisienne. Afin que le tourisme intègre la nuit urbaine, une réflexion sur l'adaptabilité des horaires doit être menée, à l'image des noctambus parisiens 
au départ de la place du Châtelet, dont la fréquence a été portée de 30 à $15 \mathrm{minu-}$ tes. Une plus grande flexibilité permettrait, d'une part, de répondre aux aspirations de la nouvelle clientele cible, celle des jeunes, et, d'autre part, de développer un tourisme urbain nocturne, car, pour l'heure, le tourisme urbain d'agrement reste encore, en grande partie, un tourisme diurne.

L'ouverture du métro la nuit renvoie cependant au problème de la sécuritế, pathologie de la ville et de son tourisme. La politique de l'accueil doit fortement s'en pré̉occuper. Face à l'insécuritế croissante, Paris annonce la création d'une police dụ métro et embauche 1000 agents de sécurité Paris pour la surveillance des écoles et des pares de stationnement, afin de permettre à la préfecture de redéployer sur le terrain des agents de police. Il faudrait done développer la coopération entre les divers départements de la municipalité parisienne et les forces de police.

\section{Conclusion}

La promotion de la ville s'est construite exclusivement sur son aspect intemporel. Le Paris éternel et impérial, des musées et des monuments prestigieux, ville figée le long de la Seine, doit etre aujourd' hui associé au bouillonnement permanent de lă métropole et à sa territorialité éclatée. Une étroite collaboration avec 1'agglomération urbaine associéc à la création d'une Direction du tourisme au sein de 1 'Administration parisienne, corrobore cette stratégie d'ouverture du territoire touristique et allie la nécessité de conjuguer simultanément dynamique touristique et dynamique urbaine. La cohérence géographique en matière de territoire touristique renvoie aux limites de l'armature du réseau des transports parisiens. L'élaboration de séjours supplémentail= res avec les couronnes de Paris devrait permettre de compléter, voire renouveler, l'offre touristique de la capitale et ainsi participer au processus de métropolisation touristique. Paris doit redevenir une porte de l'Europe et mettre en valeur sa spécificité latine.
La création de nouveaux clichés toutistiques doit amplifier l'image du Paris universel multiclientểles. Certaines familles qui limitent leur séjour à Disneyland Paris pourraient s"intéresser aux possibilités touristiques parisiennes, développées par le nécessaire recentrage sur les jeunes. Paris doit élargir sa clientèle et profiter de ce nouveau pole touristique qui ne doit pas faire concurrence au Paris monumental, nais s'y intégrè.

La politique touristique portée par une concurrence accrue doit tirer profit de cette double essence de l'attractivité parisienne, patrimoine et modernité. Elle doit réactualiser l'offre touristique parisienne au gout du jour et choisir entre inertie et dynamisme. Cette démarche est à la charnière entre ce que l'on pourrait appeler \& Mémoire *, qui assure la reproduction, et $\alpha$ projet $*$, qui fixe des ambitions, des finalités et assure la production. Si l'articulation entre les deux concepts est parfois difficile à cortéler, elle est nécessaire au renouvellement de l'attractivité de la capitale. L'alchimie de Paris

\section{Bibliographie}

Ascher, François (2001), Les noaveaux principes de l'urbanisme - La fir des villes n'est pas a l'onlre du jour, La Tourd'Aigues, l'Aube. Bailly, Jean-Paul, et Edith Heurgon (2001). Nouveaux rythes whains a quels ransports? La Tour d'Aigues, l'Aube.

Barçon, Claudine (1994), * Plaidoyer pour une ville réunifiée *, Cahiers Espaces, no 39 , décembre, p. 14-23.

Bellanger, François, et Bruno Marzloff (1996), Transit-les heux et les tenps de la mobilite, La Tour d'Aigues, l'Aube.

Burgel, Guy (1993), La ville aujourd hai, Paris, Hachette.

Cazes, Georges, et Françoise Potier (1998), Le tourisme et la ville ; expériences europénes, Paris, L'Harmattan.

Cazes, Georges, et Françoise Potier (1996), Le tourisme arbain, Paris, Presses universitaines de France, collection Que sais-je? no 3191.

Galléty, Jean-Claude, et Mariè-Thérèse Kolaczko, (dir) (2001), Les temps de la wille et les nodes de vie, Lyon, Certu.

Ingallina, Patrizia (2001), Le projet urbain, Paris, Presses universilaires de France, collection Que sais-je? no 3585 . trouvera un nouvel essor dans cette concurrence porteuse d'évolution.

Édith Fagnoni est maîte de conference a I'Vnstifut Universitaire de Formation des Mattes de Paris : membre du Laboratoine I.S.I.S. (Ingéniếrie des Systèmes d'information stratégiques et decisionnels) de l'Université de Marne-La-Vallée - équipe Stratégie du Tourisme et Information: et associé au Laboratoire C.R.E.T.E.I.L (Centre de Recherche sur l'Espace, les Transports, l'Environnement et les Institutions Locales) de l'Univer. sité de Paris $X I I$ - equipe EPPUR (sur l'Evaluation, les Pratiques, les Projets et les Paysages Urbains).

Colette Aymard est enselgnante à l'hnstitut de Geographie - Universite Paris I et membre du GIS Socio-économique de I'Habitat (Centre National de la Recherche Scienvifique, Paris I, Institut National d'Éndes Démographiques).

Lacaze, Jean-Paul (1994), Paris, urbanisme d'Etat et destin d'une ville, Paris, Flammarion.

Lazzarotti, Olivier (1995), Du bisir sons tourisme? Conribunion a l'etude des loisirs periubains, Paris, L'Harmattan.

Origet du Cluzeau, Claude (1998), Le tourisme calfwel, Paris, Presses universitaires de France, Collection Que sais je ? no 3389.

Paquot, Thierry (dir) (2001), Le quotidien urbain, Paris, La Decouverte et Syros.

Pearce, Douglas (1998), a Tourism Development in Paris - Public Intervention $\aleph_{\text {. Annals }}$ of Tourism Research, 25 (2), p. 457-476.

Pearce, Douglas (1999), a Tourism in Paris Studies at the Microscale \&, Annals of Tourism Reseanch, 26 (1), p. 77-97,

Queige, Laurent (2001), a La Seine-SaintDenis, nouvelle frontiere du tourisme parisien s, Cahiers Espaces, no 186, octobre, p. 1620 .

Roncayolo, Matcel (1999), La ville et sesterritoires, Lagny, Gallimard, collection Folio. 